\title{
REVIEW
}

\section{Cardiovascular Risk Reduction: What Do Recent Trials with Rosuvastatin Tell Us?}

Gianna Fabbri · Aldo Pietro Maggioni

Received: April 8, 2009 / Published online: May 14, 2009 / Printed: June 8, 2009

(c) Springer Healthcare Communications 2009

\section{ABSTRACT}

Abundant evidence from large-scale clinical trials supports the importance of lowering low-density lipoprotein cholesterol (LDL-C) to decrease the risk of cardiovascular disease (CVD) events. The LDL-C targets in various guidelines remain important treatment goals but, even in trials where statin therapy achieves substantial reduction of LDL-C, a significant number of CVD events still occur and the residual risk remains high. These findings suggest that lipid parameters other than LDL-C, such as high-density lipoprotein cholesterol (HDL-C), triglycerides, and LDL particle size, can influence the risk of CVD. On this basis, other strategies that can alter the lipid profile, in particular raising HDL-C, may provide additional benefits. Other factors such as HDL-C functionality and susceptibility to oxidation and inflammatory factors can also influence cardiovascular risk. In addition to the modifications of the lipid profile, statins have cholesterolindependent beneficial pleiotropic effects. The

Gianna Fabbri · Aldo Pietro Maggioni $(\bowtie)$

ANMCO Research Center, Via La Marmora 34,

50121 Florence, Italy. Email: maggioni@anmco.it contribution of these effects to event reduction is not yet fully understood. Recently, the body of evidence has expanded to support the use of intensive statin therapy in broader patient populations. The JUPITER trial has shown the benefit of intensive statin treatment in low-risk subjects with high levels of high-sensitivity C-reactive protein and average levels of LDL-C. Unlike the setting of primary and secondary prevention, the results of statin trials in patients with heart failure have shown no clear benefit in terms of survival. The recently published AURORA trial was carried out to investigate the effect of rosuvastatin in patients with end-stage renal disease undergoing chronic hemodialysis. In this trial no benefit on cardiovascular events was shown with statin therapy. In conclusion, large outcomes trials have clearly shown that statin treatments have a favorable benefit/risk profile in a large range of patients at different levels of risk, with the exception of patients with heart failure and those with renal disease undergoing dialysis. Further evidence is needed on the role of therapeutic strategies on the so-called residual risk.

Keywords: cardiovascular risk factors; HDL-C; hypercholesterolemia; LDL-C; primary prevention; secondary prevention; statin therapy 


\section{INTRODUCTION}

Despite significant advances in the understanding of the causes and treatment of cardiovascular disease (CVD), it remains the leading cause of death worldwide. ${ }^{1,2}$ Low-density lipoprotein cholesterol (LDL-C) is one of the most studied risk factors for CVD, and it is now well established that there is a direct correlation between the levels of plasma LDL-C and the risk of coronary artery disease. ${ }^{3}$

Over the past 20 years, the results of many large-scale, randomized clinical trials have shown that use of statin therapy to lower LDL-C concentrations can significantly reduce the incidence of mortality, major coronary events, stroke, and the need for revascularization in a broad spectrum of patients (Table 1). ${ }^{4-10}$ These benefits are evident both in patients at relatively low risk, who have average cholesterol levels and no previous atherosclerotic events, as well as in those who survived an acute myocardial infarction, had elevated levels of LDL-C, and other markers of heightened risk. ${ }^{10}$ Overall, statins reduce coronary heart disease morbidity and mortality by approximately 30\%-40\%, with the magnitude of the effect being directly related to the absolute reduction in LDL-C. ${ }^{10}$ The proportional reductions in major cardiovascular events appear to be similar irrespective of age, gender, preexisting CVD, or baseline levels of LDL-C or total cholesterol (Table 2). ${ }^{10}$

In addition, data from a meta-analysis of four large clinical trials investigating high-dose statins (intensive statin therapy) versus standard statin treatment have shown a further significant benefit in patients with acute coronary events or stable coronary heart disease, and reinforced the concept that for LDL-C, "the lower the better."11 The trials analyzed were the TNT (Treating to New Targets); the IDEAL (Incremental Decrease in End Points Through Aggressive LipidLowering); the PROVE-IT-TIMI 22 (Pravastatin or Atorvastatin Evaluation and Infection Therapy Thrombolysis in Myocardial Infarction-22); and the A to Z (Aggrastat to Zocor). ${ }^{12-15}$ The metaanalysis showed a significant $16 \%$ odds reduction of coronary death or myocardial infarction (9.4\% vs. 8.0\%; OR 0.84; 95\% CI 0.77-0.91) and of coronary death or any cardiovascular event (32.3\% vs. $28.8 \%$; OR 0.84; 95\% CI 0.80-0.89) in patients allocated to the intensive statin regimen group versus those on standard therapy. The main benefit was seen in preventing nonfatal cardiovascular events such as myocardial infarction, stroke, angina, and revascularization, although there was also a favorable trend

Table 1. Reduction in the 5-year incidence of cardiovascular events per $1.0 \mathrm{mmol} / \mathrm{L}$ reduction in LDL-C: a meta-analysis of 14 randomized trials of statins. ${ }^{10}$

\begin{tabular}{lccc}
\hline & \multicolumn{3}{c}{ Events } \\
\cline { 2 - 3 } Endpoint & Treatment $(n=45,054)$ & Control $(\boldsymbol{n = 4 5 , 0 0 2})$ & RR (95\% CI) \\
\hline Any death, \% & 8.5 & 9.7 & $0.88(0.84,0.91)$ \\
Coronary heart disease death, \% & 3.4 & 4.4 & $0.81(0.76,0.85)$ \\
Nonfatal myocardial infarction, \% & 4.4 & 6.2 & $0.74(0.70,0.79)$ \\
Coronary revascularization, \% & 5.8 & 7.6 & $0.76(0.73,0.80)$ \\
Any stroke, \% & 3.0 & 3.7 & $0.83(0.78,0.88)$ \\
\hline
\end{tabular}

$\mathrm{CI}=$ confidence intervals; LDL-C=low-density lipoprotein cholesterol; $\mathrm{RR}=$ rate ratio. 
in the reduction of cardiovascular death $(3.8 \%$ vs. $3.3 \%$; OR 0.88 ; $95 \%$ CI 0.78-1.00). ${ }^{11}$

On the basis of these findings, LDL-C goals in clinical practice have been updated. In the US, the National Cholesterol Education Program (NCEP) guidelines recommend a goal of $1.8 \mathrm{mmol} / \mathrm{L}(70 \mathrm{mg} / \mathrm{dL})$ in very-high-risk patients (established CVD with diabetes, smoking, metabolic syndrome, or $\geq 2$ major risk factors), and $2.6 \mathrm{mmol} / \mathrm{L}(100 \mathrm{mg} / \mathrm{dL})$ in high- or moderately-high-risk patients. ${ }^{3}$ The recently updated European guidelines recommend a goal of $<2.5 \mathrm{mmol} / \mathrm{L}$ in high-risk patients (or $<2.0 \mathrm{mmol} / \mathrm{L}$ if feasible), and $<3.0 \mathrm{mmol} / \mathrm{L}$ for all other patients. ${ }^{16}$ However, despite the evolution of these lipid management guidelines and the availability of highly effective LDL-lowering therapies, substantial cardiovascular event rates are still observed.

\section{THE RESIDUAL CARDIOVASCULAR RISK}

Current lipid-lowering therapy only partly reduces cardiovascular risk, leaving a residual risk of approximately two-thirds of pretreatment (baseline) risk. ${ }^{17}$ Generally, the rate of major coronary events varies from $62 \%$ in primary prevention to $75 \%$ in secondary prevention trials. ${ }^{18}$ For example, in the Scandinavian Simvastatin Survival Study, therapy reduced the relative risk of coronary events by approximately $30 \%$ over 5 years, from an absolute risk of $28 \%$ to $19 \%$. The event rate in the placebo group was $5.2 \%$, and $3.8 \%$ per year in the treatment group. ${ }^{4}$ A cardiovascular events rate of $2 \%-3 \%$ per year is generally considered high, indicating that the treatment group remained at unacceptably high risk. The Heart

Table 2. Effects of statin therapy on major coronary events according to baseline factors. ${ }^{10}$

\begin{tabular}{|c|c|c|c|}
\hline \multirow[b]{2}{*}{ Groups } & \multicolumn{2}{|c|}{ Events } & \multirow[b]{2}{*}{$\mathrm{RR}(95 \% \mathrm{CI})$} \\
\hline & Treatment $(n=45,054)$ & Control $(n=45,002)$ & \\
\hline \multicolumn{4}{|l|}{ Age, years, $\%$} \\
\hline$\leq 65$ & 6.1 & 8.5 & $0.74(0.69,0.79)$ \\
\hline$>65$ & 9.5 & 11.9 & $0.81(0.76,0.88)$ \\
\hline \multicolumn{4}{|c|}{ Total cholesterol, mmol/L, \% } \\
\hline$\leq 3.5$ & 6.8 & 8.7 & $0.76(0.68,0.84)$ \\
\hline$>3.5-4.5$ & 7.3 & 9.6 & $0.77(0.71,0.83)$ \\
\hline$>4.5$ & 9.3 & 12.9 & $0.78(0.72,0.85)$ \\
\hline \multicolumn{4}{|l|}{ Previous disease } \\
\hline Prior MI, \% & 11.7 & 15.4 & $0.78(0.74,0.84)$ \\
\hline Other CHD, \% & 8.7 & 11.4 & $0.77(0.68,0.87)$ \\
\hline None, $\%$ & 4.5 & 6.1 & $0.72(0.66,0.80)$ \\
\hline \multicolumn{4}{|l|}{ Gender, \% } \\
\hline Male & 7.8 & 10.6 & $0.76(0.72,0.80)$ \\
\hline Female & 6.1 & 7.3 & $0.82(0.73,0.93)$ \\
\hline
\end{tabular}

$\mathrm{CHD}=$ coronary heart disease $\mathrm{CI}=$ confidence intervals; $\mathrm{MI}=$ myocardial infarction; $\mathrm{RR}=$ rate ratio. 
Protection Study (HPS), enrolling more than 20,000 patients, had sufficient power to examine the effect of treatment with simvastatin on a variety of cardiovascular outcomes in a broad range of individuals with different risk profiles. In this study, patients receiving simvastatin had a $19.8 \%$ cardiovascular event rate compared to a $22.3 \%$ event rate in control group, which equates to a $24 \%$ relative risk reduction in those treated with a statin. ${ }^{6}$ This important risk reduction was consistent in all subgroups and across all stratifications of baseline cholesterol levels, even those with LDL-C lower than $3.0 \mathrm{mmol} / \mathrm{L}$. However, despite this impressive result, it is evident that $76 \%$ of the events were not prevented, with the residual risk being highest in diabetic patients with coronary artery disease. ${ }^{19} \mathrm{~A}$ similar finding was also observed in other trials such as CARE, PROSPER, and LIPID. 5,20,21

It may be that this residual risk results from starting statin therapy too late or at too low a dose, or that the treatment period in clinical trials is not long enough to observe the full range of beneficial effects. ${ }^{18}$ In order to address this residual cardiovascular risk and further reduce the rate of cardiovascular events, it will be necessary to gain a greater understanding of the contributory role of other known risk factors in CVD and to understand the potential that the nonlipid effects of statins may offer, as well as continuing to investigate the value of lower LDL-C goals.

\section{RISK FACTORS FOR CARDIOVASCULAR DISEASE}

The search for additional cardiovascular risk factors is constantly striving to improve physicians' ability to accurately assess the risk of cardiovascular events and to provide the most appropriate care. A number of physiologic and behavioral risk factors for CVD are known. Non- modifiable risk factors include advanced age, male gender, a family history of CVD, and genetic conditions such as familial hypercholesterolemia. Of the modifiable risk factors, metabolic syndrome, cigarette smoking, and elevated high-sensitivity C-reactive protein (hsCRP) levels predict a high residual risk. Lifestyle modifications, such as diet and exercise, continue to have an important role in the primary prevention of CVD, particularly in obese subjects and those with the metabolic syndrome. ${ }^{22-27}$ Diabetes mellitus is also a strong predictor of adverse cardiovascular outcomes, and treatment with a statin is recommended for all patients with this condition.

In the last decade the relationship between lipids, inflammation, and their effect on the arterial wall has been the subject of considerable interest. ${ }^{28}$ Risk factors for atherogenesis give rise to a variety of stimuli which activate the inflammatory cascade; and every step in atherogenesis is believed to involve cytokines, other bioactive molecules, and cells that are characteristic of inflammation. These pathophysiologic insights provide the biological plausibility for the potential use of inflammatory markers as indicators or predictors of atherosclerotic disease. HsCRP is a marker of inflammation that has been shown to predict cardiovascular risk independent of major "traditional" risk factors such as smoking, hypertension, diabetes, and cholesterol levels. ${ }^{29,30}$ Several studies have demonstrated the ability of hsCRP to increase the predictive capacity of other risk factors. ${ }^{31,32}$ Evidence for a relationship between a patient's baseline level of hsCRP and future vascular risk has been increasingly demonstrated in studies from the USA and Europe, among women as well as men, elderly and middle-age subjects, smokers and nonsmokers, and among those with or without diabetes. ${ }^{33}$ HsCRP levels seem to have both short- and long-term predictive value. ${ }^{34,35}$ Treatment with statins lowers 
the concentration of hsCRP. ${ }^{36,37}$ Several studies carried out in patients with stable coronary disease, acute coronary syndrome, or healthy persons, have shown that patients with lower hsCRP levels have a more favorable clinical outcome than those with higher hsCRP levels, regardless of the level of LDL-C. ${ }^{36,38-42}$

Multiple studies have also established that a low level of high-density lipoprotein cholesterol (HDL-C) is an independent risk factor for CVD. ${ }^{43-45}$ It has been shown that each $1 \mathrm{mg} / \mathrm{dL}$ decrease in HDL-C is associated with a $2 \%-3 \%$ higher risk of coronary death independent of LDL-C level. ${ }^{43}$ Low serum levels of HDL-C are likely associated with an increased cardiovascular risk because they reflect an increase in atherogenic lipoproteins. In addition to its major role in reverse cholesterol transport, HDL-C has many other biological activities that may contribute to its protective effect atherosclerosis including antioxidant, antinflammatory, antithrombotic/profibrinolytic (reducing platelet aggregation and coagulation), and vasoprotective (facilitating vascular relaxation and inhibiting leukocyte chemotaxis and adhesion) activity. ${ }^{44,46}$ There is some evidence to support this hypothesis from both experimental models of atherosclerosis and clinical trials. ${ }^{47,48}$ Therefore, low HDL-C could be considered a potential therapeutic target, in addition to aggressively lowered LDL-C, for the reduction of residual cardiovascular risk. ${ }^{49-52}$

First-line therapy of low HDL-C usually consists of nonpharmacological interventions such as improved fitness, smoking cessation, dietary changes, and weight loss. If these measures are unsuccessful, pharmacological therapies such as niacin, fibrates, thiazolidinediones, or bile acid sequestrants may be initiated. ${ }^{53}$

Recent studies have investigated the inhibition of cholesteryl ester transfer protein (CETP) as a potential pharmacological approach for increasing HDL-C levels. Torcetrapib was the most advanced CETP inhibitor in development. However, in the ILLUSTRATE trial (Investigation of Lipid Management Using Coronary Ultrasound to Assess Reduction of Atherosclerosis by CETP Inhibitors and HDL Elevation), the combination of torcetrapib and atorvastatin was not superior to atorvastatin alone in reducing atherosclerotic coronary plaque burden. ${ }^{54}$ The results of two double-blind, randomized trials in patients with heterozygous familial hypercholesterolemia (RADIANCE 1) or mixed dyslipidemia (RADIANCE 2) using torcetrapib and atorvastatin in combination, also showed no changes in the progression of carotid intima media thickness (CIMT) compared with the results of atorvastatin alone. ${ }^{55,56}$ Finally, a randomized, double-blind trial involving 15,067 patients at high cardiovascular risk (the ILLUMINATE trial) was terminated prematurely because patients allocated to the torcetrapib/atorvastatin combination arm showed an increased risk of mortality and morbidity compared with those treated with atorvastatin alone. ${ }^{57}$

These findings suggest that the functionality of HDL may be more important than HDL-C levels themselves and that the relationship between HDL-C and cardiovascular risk is more complex than expected. $.^{58,59} \mathrm{HDL}-\mathrm{C}$ functionality (ie, the ability to perform its atheroprotective function) is partly independent of its concentration. Many factors can affect HDL-C functionality: modifications of HDL proteins and lipids such as advanced glycation and oxidation, changes in HDL-C composition and size of particles, and changes in the abundance of various proteins and lipids carried by HDL-C. ${ }^{58}$ A single common denominator reflecting the multiple HDL functions is yet to be found, preventing the direct measurement of each HDL-C function as the way to assess the atheroprotective capacity of HDL-C. 
Despite the highly publicized failure of torcetrapib, the role of CETP inhibitors is still under evaluation to assess whether the off-target effects are a mechanism- or molecule-related toxicity. In addition, alternative therapeutic strategies for improving the functionality of HDL are being investigated. ${ }^{60}$

\section{MODIFICATION OF CARDIOVASCULAR RISK FACTORS WITH ROSUVASTATIN}

\section{Effects on Atherogenic Lipid Profile}

Lipoproteins other than LDL-C can influence the development of coronary atherosclerosis and its response to therapy. Important components of atherogenic lipids are triglycerides, low HDL-C, and small dense LDL. In the STELLAR trial (Statin Therapies for Elevated Lipid Levels Compared Across Doses to Rosuvastatin), ${ }^{61}$ the largest trial comparing the lipid-modifying efficacy of statins, the greater efficacy of rosuvastatin in reducing LDL-C compared with atorvastatin, simvastatin, and pravastatin across dose ranges has been shown. In this 6-week trial, 2431 patients with hypercholesterolemia were randomized to different dosages of the four statins; $94 \%$ of patients completed the study. There was a significantly greater LDL-C reduction achieved with rosuvastatin $10 \mathrm{mg}$ compared with atorvastatin $10 \mathrm{mg}$ (\% change -45.8 vs. $-36.8 ; P<0.001)$, rosuvastatin $20 \mathrm{mg}$ reduced LDL-C significantly more than atorvastatin 20 and $40 \mathrm{mg}$ (\% change -52.4 vs. -42.6 and -47.8 ; $P<0.001$ and $P<0.002$, respectively) and rosuvastatin $40 \mathrm{mg}$ reduced LDL-C more than atorvastatin $40 \mathrm{mg}$ (\% change -55.0 vs. $-47.8 ; P<0.001)$.

Rosuvastatin also produced a significantly greater reduction in total cholesterol, similar or better reduction in triglycerides, and increase in HDL-C. In Table 3, the increase in HDL-C across dose ranges is shown; as rosuvastatin doses are increased, HDL-C changes from baseline increase while atorvastatin produced smaller percentage increases in HDL-C. ${ }^{61}$ The rate of adverse events was similar among groups and ranged from $40 \%$ to $56 \%$ per group, being $46 \%$ in the total study population. Twenty-nine patients had serious adverse events; the number per group ranged from 0 (rosuvastatin $40 \mathrm{mg}$ ) to 5 (simvastatin $40 \mathrm{mg}$ ). The other adverse events reported were mild and similar across groups, as were changes in clinical laboratory results. The number of reported adverse events tended to be highest with higher doses. The most common adverse events were pain, pharyngitis, myalgia, and headache.

These results are supported by data presented from the MERCURY I study (Measuring Effective

Table 3. Increase in HDL-C from baseline across dose ranges of statins: results from the STELLAR study. ${ }^{61}$

\begin{tabular}{lcccc}
\hline & \multicolumn{4}{c}{ HDL-C change, \% } \\
\cline { 2 - 5 } Dose & Rosuvastatin & Atorvastatin & Simvastatin & Pravastatin \\
\hline $10 \mathrm{mg}$ & $7.7^{*}$ & 5.7 & 5.3 & 3.2 \\
$20 \mathrm{mg}$ & $9.5^{*}$ & 4.8 & 6.0 & 4.4 \\
$40 \mathrm{mg}$ & $9.6^{*}$ & 4.4 & 5.2 & 5.6 \\
$80 \mathrm{mg}$ & $\mathrm{NA}$ & 2.1 & 6.8 & $\mathrm{NA}$ \\
\hline
\end{tabular}

*Significantly different for all dose ranges.

HDL-C=high-density lipoprotein cholesterol; NA=not available. 
Reductions in Cholesterol Using Rosuvastatin Therapy), showing that patients who switched to rosuvastatin $10 \mathrm{mg}$ had a significantly greater improvement in their lipid parameters than those who remained on atorvastatin $10 \mathrm{mg}$, simvastatin $20 \mathrm{mg}$, or pravastatin $40 \mathrm{mg} .{ }^{62}$ The proportion of patients who achieved the Joint European Societies' LDL-C goal $(<116$ mg/dL) was also significantly greater for patients who switched to rosuvastatin $10 \mathrm{mg}$ compared with patients who continued to receive atorvastatin, simvastatin, or pravastatin. ${ }^{62}$ MERCURY I was a 16-week, randomized, open-label, parallel-group, switching study that compared the efficacy of rosuvastatin versus atorvastatin, simvastatin, and pravastatin in patients with type IIa or IIb hypercholesterolemia and established atherosclerosis, coronary heart disease, or type 2 diabetes.

Statins generally induce modest increases in HDL-C (5\%-15\%). However, it has not been established if this increase translates into a clinical benefit, as statins are used primarily to decrease LDL-C while other therapies, usually in combination with statins, have been used to increase HDL-C. ${ }^{63,64}$

\section{Effects on the Progression of Atheroscleroic Plaques}

In order to provide more effective disease prevention and address the residual risk of CVD, it is important to understand the mechanisms by which LDL-lowering therapies achieve their beneficial effects on atherosclerotic plaques. Therefore, imaging techniques such as intravascular ultrasound (IVUS) and measurement of CIMT using B-mode ultrasound are increasingly being used in surrogate endpoint trials to understand the relationship between levels of LDL-C and disease progression. ${ }^{65-69}$ In addition to IVUS and CIMT methodologies, other atherosclerosis imaging techniques include quantitative coronary angiography (QCA), carotid magnetic resonance imaging (MRI), and computed tomography coronary angiography, each with its own inherent advantages and disadvantages.

An imaging surrogate biomarker for atherosclerosis needs to measure changes in plaque volume/burden, and/or measure changes in plaque composition, be easily reproducible and repeatable, and correlate with clinical outcome. For many years, QCA was the standard imaging tool for the assessment and characterization of obstructed coronary arteries. ${ }^{65}$ QCA allows measurement of changes in the lumen of the vessel, and provides information throughout the coronary tree, including branch vessels and distal stenosis. QCA was used in several of the early clinical trials of statin therapy, showing a direct relationship between LDL-C levels and the rate of disease progression. ${ }^{70}$ However, it is an invasive procedure and because it is limited to providing dimensions of the lumen only, not the vessel wall, it is therefore unable to fully describe the effects of statin therapy on the plaque burden. ${ }^{71}$

IVUS is able to generate high-resolution tomographic images of the entire vessel wall as well as of the lumen, and has become the most precise method for the determination of coronary atheroma burden. IVUS allows accurate measurement of atheroma volume and evaluation of changes in atheroma burden during treatment. ${ }^{65}$ However, it is also an invasive technique, and composition of the atherosclerotic plaque can be difficult to assess.

Previous studies using IVUS imaging have shown that intensive statin therapy is more effective than moderate therapy in reducing the progression of atherosclerosis. Results from the REVERSAL study (Reversing Atherosclerosis with Aggressive Lipid lowering), a randomized study comparing the effects of pravastatin 40 $\mathrm{mg}$ /day versus atorvastatin $80 \mathrm{mg} /$ day, provide 
further evidence for the beneficial effects of intensive lipid lowering. ${ }^{72}$ In this study, 502 patients had evaluable IVUS examinations both at baseline and after 18 months of follow-up. For all IVUS measures, progression of atherosclerosis occurred in the moderate treatment cohort while plaque volume was unchanged in the intensive arm, indicating absence of progression.

In the ASTEROID study (A Study to Evaluate the Effect of Rosuvastatin On Intravascular Ultrasound Derived Coronary Atheroma Burden), a significant regression of coronary atherosclerosis was seen with rosuvastatin $40 \mathrm{mg} /$ day in patients with coronary heart disease. ${ }^{73}$ ASTEROID was a prospective, open-label, blinded endpoint trial; 507 patients had an IVUS baseline examination, and after 24 months 349 had evaluable serial IVUS examinations. In this study, the 53\% reduction in LDL-C and the $15 \%$ increase in HDL-C was associated with an IVUS-determined regression of $-1.0 \%$ for atheroma volume. ${ }^{73}$ In this trial, 379 patients also had baseline angiograms. Of these, 292 had $\geq 1$ segment with $\geq 25 \%$ diameter stenosis permitting measurement of the change in the severity of stenosis by QCA during follow-up. The lipid data for the QCA group were similar to the results for the full ASTEROID intention-totreat group. ${ }^{74}$ The relationship between changes in HDL-C and changes in percentage diameter stenosis or minimum lumen diameter in serial coronary angiographic studies of statin therapy are reported in Figure $1 .{ }^{74}$ Using the criterion of $\geq 10 \%$ change in percent-diameter stenosis for regression, $7.5 \%$ of patients showed regression whereas $89.4 \%$ showed $<10 \%$ change and $3.1 \%$ showed progression. ${ }^{74}$ This angiographic subanalysis of ASTEROID provided the first evidence that statin monotherapy, with a significant impact on both LDL-C and HDL-C, can promote regression of atherosclerosis. ${ }^{65}$
A subsequent pooled analysis of IVUS data from ASTEROID and REVERSAL, as well as the CAMELOT (Comparison of Amlodipine vs. Enalapril to Limit Occurrences of Thrombosis) and ACTIVATE (ACAT Intravascular Atherosclerosis Treatment Evaluation) studies, demonstrated that increasing HDL-C levels by $>7.5 \%$ was an independent predictor of a beneficial effect of statin therapy on plaque progression. ${ }^{75}$ Substantial atheroma regression ( $\geq 5 \%$ reduction in atheroma volume) was observed in patients with levels of LDL-C less than the mean $(87.5 \mathrm{mg} / \mathrm{dL})$ during treatment and percentage increases of HDL-C greater than the mean $(7.5 \%, P<0.001)$. These findings highlighted the importance of both reducing atherogenic lipid levels and raising HDL-C, but supporting evidence from outcomes studies is required to determine whether these changes translate into a reduction in cardiovascular events. ${ }^{75}$

B-mode ultrasound measurement of CIMT is a noninvasive tool to measure thickening of the intima media layer of the carotid and femoral arteries. ${ }^{65,71}$ Increased CIMT is associated with an increased risk of cardiovascular events and, as with QCA, there is a direct relationship between the extent of LDL-C lowering and rate of CIMT progression. ${ }^{76}$ Furthermore, CIMT regression has been observed in studies achieving intensive LDL-C lowering using highdose atorvastatin. ${ }^{77,78}$

The METEOR study (Measuring Effects on Intima-Media Thickness: an Evaluation of Rosuvastatin) used CIMT assessment to investigate the effect of rosuvastatin $40 \mathrm{mg} /$ day in patients with subclinical atherosclerosis, moderately elevated cholesterol levels, and a low risk of CVD. ${ }^{79}$ In this randomized, double-blind, placebocontrolled study, the primary endpoint was the annualized rate of change in maximum CIMT. In these low-risk patients (Framingham 10-year risk $<10 \%)$ with no established atherosclerosis and 
moderately raised LDL-C levels, rosuvastatin significantly reduced the rate of progression of maximum CIMT over 2 years compared with placebo. Rosuvastatin $40 \mathrm{mg}$ resulted in a $0.0014 \mathrm{~mm} /$ year decrease in the mean maximum CIMT compared with a progression of $0.0131 \mathrm{~mm} /$ year in placebo recipients $(P<0.0001) .{ }^{79}$ Rosuvastatin was also associated with a $49 \%$ reduction in LDL-C and an $8 \%$ increase in HDL-C levels.

These results further extended the findings of the ASTEROID study, which had included high-risk patients with established coronary artery disease, and represented the first time that a positive effect on the progression of atherosclerosis had been demonstrated, even in subjects with early signs of carotid artery disease and at low risk of coronary heart disease who would not usually be candidates for statin therapy. The findings also suggested that CIMT might be a useful tool to identify patients most likely to benefit from early implementation of statin treatment, although the value of

Figure 1. Relationship between either on-treatment level (A \& C) or percentage change in HDL-C (B \& D) and either change in percentage diameter stenosis $(\mathbf{A} \& \mathbf{B})$ or minimum lumen diameter $(\mathbf{C} \& \mathbf{D})$ in serial coronary angiographic studies of statin therapy. ${ }^{74}$ ASTEROID $=$ A Study to Evaluate the Effect of Rosuvastatin on Intravascular Ultrasound-Derived Coronary Atheroma Burden; CCAIT =Canadian Coronary Atherosclerosis Intervention Trial; HDL-C=high-density lipoprotein cholesterol; LCAS=Lipoprotein and Coronary Atherosclerosis Study; MAAS=Multicenter Anti-Atheroma Study; MARS=Monitored Atherosclerosis Regression Study; $M L D=$ minimum lumen diameter; PLAC=Pravastatin Limitation Of Atherosclerosis In The Coronary Arteries; REGRESS=Regression Growth Evaluation Statin Study.

Reproduced from Ballantyne CM, et al. ASTEROID Investigators. Effect of rosuvastatin therapy on coronary artery stenoses assessed by quantitative coronary angiography: a study to evaluate the effect of rosuvastatin on intravascular ultrasoundderived coronary atheroma burden. Circulation. 2008;117:2458-2466. With permission from Wolters Kluwer Health.

(A)

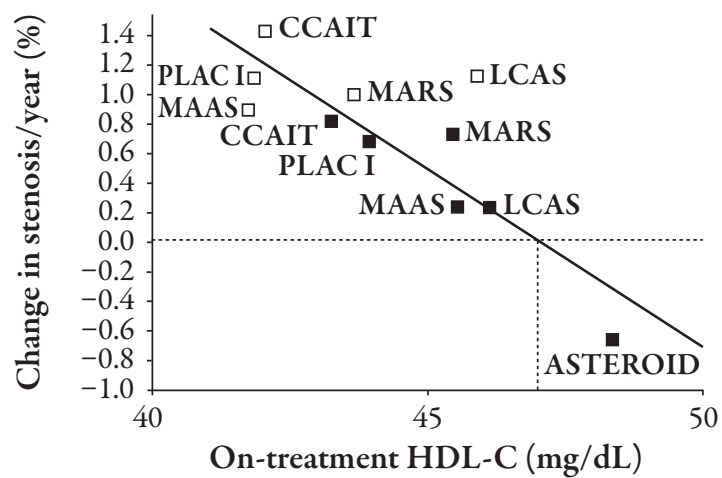

(C)

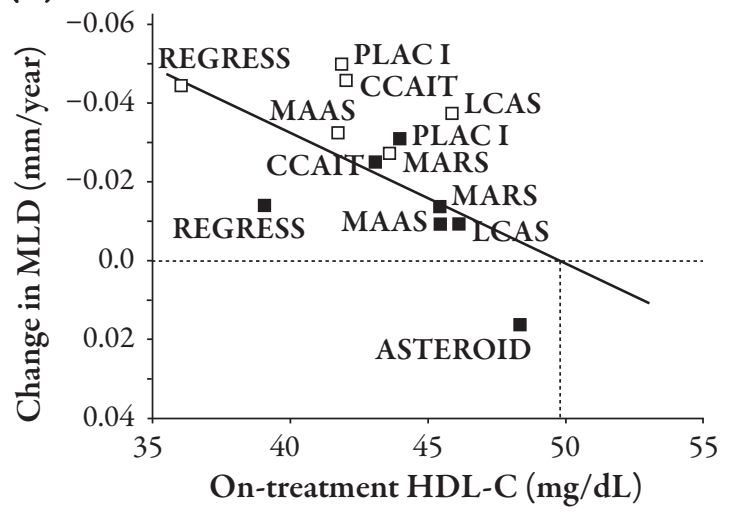

(B)

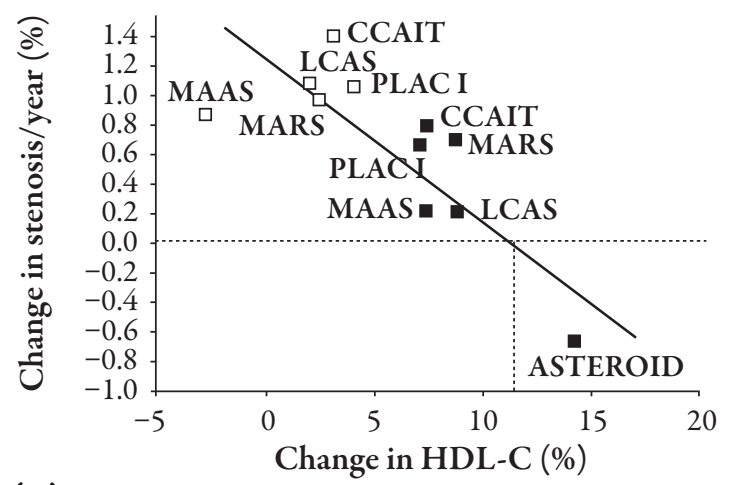

(D)

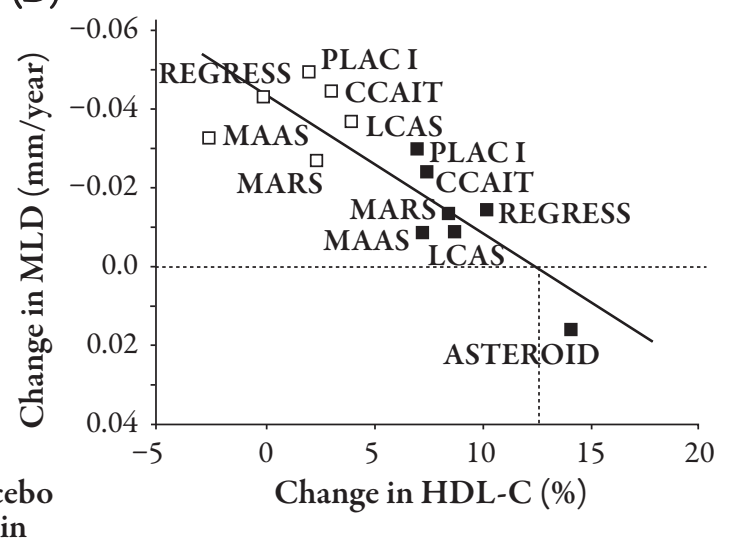


this screening approach needs to be validated in large, prospective studies.

Results from ASTEROID, METEOR, REVERSAL, and other studies confirm that intensive lipid management can regress or slow the progression of coronary atherosclerosis. To further characterize the effects of rosuvastatin on atherosclerosis, an ongoing trial, SATURN (Study of Coronary Atheroma by Intravascular Ultrasound: Effect of Rosuvastatin Versus Atorvastatin), will compare the effects of rosuvastatin $40 \mathrm{mg}$ daily with atorvastatin $80 \mathrm{mg}$ daily on atheroma volume as measured by IVUS imaging in patients with coronary artery disease. ${ }^{80}$ This is a phase 4, multicenter, double-blind, parallel-group study with 1300 patients enrolled and 2 years of follow-up. Atherosclerosis progression will be assessed in a matched segment of a single coronary artery at baseline and at the end of the treatment. The SATURN study will compare the effects of rosuvastatin and atorvastatin on the progression of coronary atherosclerosis, and provide a better understanding of the impact of intensive statin treatment on levels of LDL-C, HDL-C, and inflammatory markers on the prognosis of atherosclerosis. ${ }^{80}$

The finding that increases in HDL-C in statintreated patients may play a significant role in the promotion of atheroma regression should be considered in the selection of therapy and management of atherosclerotic artery disease. Both rosuvastatin and atorvastatin substantially reduce LDL-C but the increases of HDL-C produced by rosuvastatin are maintained across all dose ranges, whereas the effect on HDL-C of atorvastatin reaches a plateau with increasing dose. ${ }^{61}$

\section{Effects on Inflammatory Markers}

Measuring hsCRP may help to identify subjects with LDL-C levels below current treatment thresholds but who are at increased risk of future cardiovascular events and may therefore benefit from statin therapy. The JUPITER trial (Justification for the Use of statins in Prevention: an Intervention Trial Evaluating Rosuvastatin) directly addressed whether apparently healthy persons with levels of LDL-C $\leq 3.5$ $\mathrm{mmol} / \mathrm{L}$ but with elevated hsCRP $(\geq 2.0 \mathrm{mg} / \mathrm{L})$ might benefit from statin therapy in terms of first major cardiovascular event (cardiovascular death, nonfatal myocardial infarction, nonfatal stroke, hospitalization for unstable angina, and arterial revascularization procedure) ${ }^{81}$ Patients $(n=17,802)$ were randomly assigned to receive rosuvastatin $20 \mathrm{mg}$ daily or matching placebo.

This event-driven trial was prematurely stopped due to a clear treatment benefit, after a median follow-up period of 1.9 years (maximum 5.0 years). Table 4 shows the baseline and final values of LDL-C and hsCRP observed in the trial. ${ }^{81}$ Rosuvastatin treatment provided a significant reduction in the rate of the primary endpoint and the majority of its individual components (Table 5). ${ }^{81}$ The risk reduction was nearly double that observed in previous trials, in which raised LDL-C levels were used as the enrolment criteria. The extent of risk reduction can also be correlated with the intensive lowering of LDL-C levels, which were reduced by $50 \%$, compared with previous trials (Figure 2). In terms of absolute benefits, the proportion of patients meeting the primary endpoint was $1.6 \%$ in the rosuvastatin arm and $2.8 \%$ in the placebo arm with an absolute risk reduction of $1.2 \%$; the proportion of patients with a cardiac event was $0.9 \%$ in the rosuvastatin arm and $1.8 \%$ in the placebo arm, with an absolute risk reduction of $0.9 \%$. The relative risk reductions were consistent in each subgroup according to age, race, status with regard to traditional risk factors, and level of risk.

In a prespecified subanalysis of the JUPITER study, the effects of lowering both LDL-C and 
Table 4. JUPITER study: LDL-C and hsCRP values. ${ }^{81}$

\begin{tabular}{|c|c|c|c|c|c|}
\hline & \multicolumn{2}{|c|}{ Baseline } & \multicolumn{2}{|c|}{48 months } & \multirow[b]{2}{*}{$P$} \\
\hline & $\begin{array}{c}\text { Rosuvastatin } \\
(n=8901)\end{array}$ & $\begin{array}{l}\text { Placebo } \\
(n=8901)\end{array}$ & Rosuvastatin* & Placebo $\dagger$ & \\
\hline Median LDL-C, mg/dL & 108 & 108 & 55 & 109 & $<0.001$ \\
\hline Median hsCRP, mg/L & 4.2 & 4.3 & 1.8 & 3.3 & $<0.001$ \\
\hline
\end{tabular}

*Patient numbers at 48 months for rosuvastatin arm: LDL-C $n=440$, hsCRP $n=439$.

†Patient numbers at 48 months for placebo arm: LDL-C $n=414$, hsCRP $n=407$.

hsCRP=high-sensitivity C-reactive protein; LDL-C=low-density lipoprotein cholesterol.

Table 5. JUPITER study results: primary endpoint and its components. ${ }^{81}$

Reproduced from: Ridker PM, et al. N Engl J Med. 2008;359:2195-2207. Copyright @ 2008 Massachusetts Medical Society. All rights reserved.

\begin{tabular}{lcccccc}
\hline & Rosuvastatin $(n=8901)$ & \multicolumn{2}{c}{ Placebo $(n=8901)$} & & \\
\cline { 2 - 5 } & $n$ & $\begin{array}{c}\text { Rate per 100 } \\
\text { persons-year }\end{array}$ & $n$ & $\begin{array}{c}\text { Rate per 100 } \\
\text { persons-year }\end{array}$ & HR (95\% CI $)$ & $P$ \\
\hline Primary endpoint* & 142 & 0.77 & 251 & 1.36 & $0.56(0.46,0.69)$ & $<0.00001$ \\
Nonfatal MI & 22 & 0.12 & 62 & 0.33 & $0.35(0.22,0.58)$ & $<0.00001$ \\
Nonfatal stroke & 30 & 0.16 & 58 & 0.31 & $0.52(0.33,0.80)$ & 0.003 \\
Arterial revascularization & 71 & 0.38 & 131 & 0.71 & $0.54(0.41,0.72)$ & $<0.00001$ \\
Hospitalization for unstable & 16 & 0.09 & 27 & 0.14 & $0.59(0.32,1.10)$ & 0.09 \\
angina & 198 & 1.0 & 247 & 1.25 & $0.80(0.67,0.97)$ & 0.02 \\
Any death & & & & & \\
\hline
\end{tabular}

*Nonfatal MI, nonfatal stroke, hospitalization for unstable angina, revascularization, death from cardiovascular causes. $\mathrm{CI}=$ confidence intervals; $\mathrm{MI}=$ myocardial infarction; $\mathrm{HR}=$ hazard ratio .

hsCRP levels were evaluated. ${ }^{82}$ Patients treated with rosuvastatin who achieved LDL-C levels $<1.8 \mathrm{mmol} / \mathrm{L}$ experienced a $55 \%$ reduction in vascular events compared with placebo. A $62 \%$ reduction in event rate was also observed for rosuvastatin recipients who achieved hsCRP levels $<2 \mathrm{mg} / \mathrm{L}$. In patients treated with rosuvastatin who achieved both these levels, the reduction in vascular events was $65 \%$ compared with a $33 \%$ reduction in patients who achieved one or neither target. ${ }^{82}$ These analyses provide further insight into the mechanisms by which statins reduce cardiovascular risk and the role of their anti-inflammatory properties. In addition, rosuvastatin significantly reduced the incidence of symptomatic venous thromboembolism, an effect independent of its benefit in reducing the risk of arterial thrombosis. ${ }^{83}$

Rosuvastatin was generally well tolerated in this study, although there was an increased incidence of physician-reported diabetes. However, no significant difference in fasting blood glucose levels or glycosuria was observed between the two study groups during the follow-up 
Figure 2. Primary endpoint data* from major placebo-controlled statin outcomes studies correlating risk reduction with LDL-C reduction. The area of the plotted symbol is proportional to the number of patients in the study. ${ }^{*}$ Primary endpoint used for all studies, with the exception of $4 S$ (major coronary events), HPS (major vascular event), LIPID (CHD death or nonfatal MI), SPARCL (major cardiovascular event). 4S=Scandinavian Simvastatin Survival Study; AF/TEX=Air Force/Texas Coronary Atherosclerosis Prevention Study; ALERT=Assessment of Lescol in Renal Transplantation; ASCOT=Anglo-Scandinavian Cardiac Outcomes Trial; ASPEN=Atorvastatin Study for the Prevention of coronary heart disease End points in Non-insulin-dependent diabetes mellitus; CARDS=Collaborative AtoRvastatin Diabetes Study; $\mathrm{CARE}=$ Cholesterol And Recurrent Events; HPS=Heart Protection Study; JUPITER=Justification for the Use of statins in Prevention: an Intervention Trial Evaluating Rosuvastatin; LDC-L=low-density protein cholesterol; LIPID=Long-term Intervention with Pravastatin in Ischemic Disease; LIPS=Lescol Intervention Prevention Study; PROSPER=PROspective Study of Pravastatin in the Elderly at Risk; SPARCL=Stroke Prevention by Aggressive Reduction in Cholesterol Levels; WOSCOPS=West Of Scotland COronary Prevention Study.

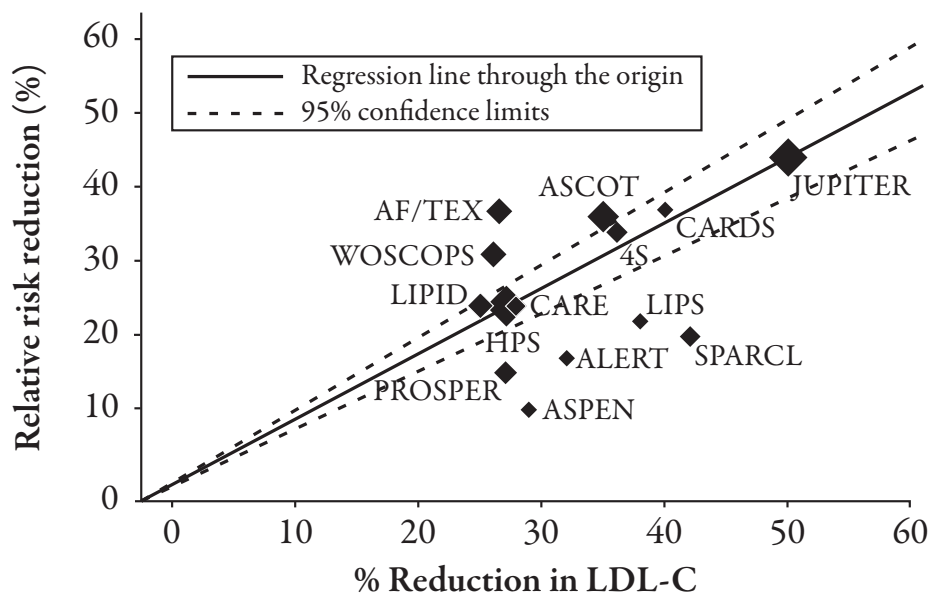

period. There were also no significant differences between the two groups with regard to muscle weakness, newly diagnosed cancer, or disorders of the hematologic, gastrointestinal, hepatic, or renal systems. ${ }^{81}$

These findings confirm that hsCRP is a significant independent predictor of CVD risk and raise important questions regarding the wider use of statins, and choice of statin, in primary prevention. Since half of all vascular events occur in patients with normal or low LDL-C levels, identification of new screening tools to further reduce cardiovascular risk is essential. In the editorial accompanying the JUPITER publication, two questions are highlighted: how to use hsCRP measurements in daily practice, and if the indication for statin treatment should be expanded. ${ }^{84}$ In JUPITER, the information on the role of hsCRP is limited by the fact that the trial did not compare subjects with and without high levels of hsCRP. The use of more widespread screening of hsCRP values must also take into account both the variability and the elevation that occurs with infections; abnormallyhigh CRP levels do not always reflect CVD. However, the results of the JUPITER study are a step forward in the field of primary prevention, even if it is important to take a careful look at the evidence and see how it should best be integrated into the existing guidelines. ${ }^{85}$ The scarcity of long-term safety data for lowering LDL-C in low-risk subjects suggests a careful evaluation before prescribing a statin to healthy asymptomatic individuals for the rest of their lives. Nevertheless, it is likely that clinical guidelines will be revisited in light of these findings in order to assist physicians in identifying new patients most likely to benefit from statin therapy. 
BEYOND

HYPERCHOLESTEROLEMIA: HEART FAILURE AND END-STAGE RENAL DISEASE

Although the benefits of statin therapy have been demonstrated in an increasing range of patients, both in primary and secondary prevention, there remain several groups at risk of cardiovascular events for whom statins are not yet indicated. In addition to lipid-lowering effects, experimental data indicate that rosuvastatin may also exhibit a wide variety of actions such as improvement in endothelial function, reduction of vascular inflammatory processes, antioxidation, and prevention of cardiac arrhythmias. ${ }^{86,87}$ Most of these effects may target important components of the pathophysiology of heart failure, a condition where mortality remains high despite the widespread use of recommended treatments such as angiotensin-converting enzyme inhibitors and beta-blockers. ${ }^{88}$ Data from observational studies and post-hoc analyses of randomized clinical trials indicate that patients with chronic heart failure receiving statin treatment had favorable cardiovascular outcomes both in ischemic and nonischemic cardiomyopathy. ${ }^{89,90}$

Recently, two large randomized trials investigating statin therapy in patients with heart failure have been published. In the CORONA trial (Controlled Rosuvastatin Multinational Trial in Heart Failure), conducted in 5011 patients with systolic heart failure due to ischemic etiology, rosuvastatin $10 \mathrm{mg} /$ day reduced LDL-C and hsCRP levels but did not provide a benefit in terms of cardiovascular deaths, heart attacks, or strokes over a follow-up period of approximately 3 years. ${ }^{91}$ However, a highly significant reduction in cardiovascular hospitalizations in older patients with advanced systolic heart failure was observed, and based on this, guidelines from the European Heart Association recommend statin treatment for reducing cardiovascular hospitalizations in patients with systolic heart failure of ischemic origin. ${ }^{92}$

The GISSI Heart Failure trial (GISSI-HF), which involved 4594 adults of all ages with chronic heart failure of any etiology, also failed to show any benefit in clinical outcome (allcause death and hospitalizations for cardiovascular causes) after nearly 4 years of treatment with rosuvastatin $10 \mathrm{mg}$ daily. ${ }^{93}$ The results of CORONA and GISSI-HF reinforce the idea that benefit from statin treatment appears, primarily, to derive from the prevention of coronary artery disease; in clinical conditions where coronary disease does not significantly contribute to the causes of death, statins seem to be less effective. Furthermore, findings in populations without heart failure cannot be extrapolated to patients with heart failure. Once heart failure is established, statins may not improve morbidity/ mortality of these patients. ${ }^{94}$ Since the effect of statin withdrawal in ischemic heart failure is not yet known, current statin treatment should not be stopped in patients with heart failure on the basis of the evidence of CORONA and GISSI-HF and of the safety profile of rosuvastatin.

The role of statins is also under investigation in patients with end-stage renal disease (ESRD). In these patients, CVD is the leading cause of death. Previous studies have shown that total and cardiovascular mortality were $32 \%$ and $36 \%$ lower, respectively, among ESRD patients receiving statins compared with those not receiving them..${ }^{95}$ However, the recent $4 \mathrm{D}$ study has shown no improvement in mortality from statin treatment in patients with diabetes and ESRD receiving hemodialysis. ${ }^{96}$

The recently completed AURORA trial (A Study to Evaluate the Use of Rosuvastatin in Subjects on Regular Haemodialysis: an 
Assessment of Survival and Cardiovascular Events) evaluated the effect of rosuvastatin treatment in a population of high-risk patients with ESRD. ${ }^{97}$ In this placebo-controlled study, 2776 patients undergoing maintenance hemodialysis were randomized to receive rosuvastatin $10 \mathrm{mg} /$ day or placebo. Despite a significant reduction in LDL-C and hsCRP levels, rosuvastatin treatment failed to achieve the combined primary endpoint of death from cardiovascular causes, nonfatal MI, or nonfatal stroke (HR 0.96; 95\%CI 0.84-1.11). ${ }^{98}$ Among the components of the primary endpoint, a neutral effect of rosuvastatin therapy compared with placebo was also observed. Several considerations could be done with respects to these results: first, the event rates in placebo group were lower than expected; furthermore, the trial, enrolling patients who had not been treated with statins during the previous 6 months, may have excluded those with previous cardiovascular events or at increased risk who were most likely to benefit from treatment. Finally, approximately $50 \%$ of patients in AURORA discontinued the treatment, but the per-protocol analysis confirmed the lack of an effect of rosuvastatin on the primary composite endpoint. An accompanying editorial suggested that the most likely explanation for these findings is that patients undergoing dialysis have a different causal pathway for early cardiovascular events and death compared with the general population. Therefore, the benefits of LDL reduction cannot be transferred directly from the field of primary or secondary prevention to patients with renal disease undergoing dialysis. ${ }^{99}$

\section{CONCLUSIONS}

With the widespread adoption of statin treatment, substantial progress has been made in the management of hypercholesterolemia and in reducing cardiovascular morbidity and mortality in patients at high risk of cardiovascular events in both primary and secondary prevention. Outcomes from several studies indicate a direct relation between cardiovascular event rate and LDL-C reduction, supporting the recommendation to treat patients to a definite target of LDL-C concentration. Moreover, data from observational studies indicate that a continuous positive relationship exists between blood cholesterol concentrations and coronary artery disease, suggesting that there is not a lower threshold for lowering cholesterol. ${ }^{80}$ Indeed NCEP ATP III (National Cholesterol Education Program Adult Treatment Panel III) recommendations indicate that lowering LDL-C below $70 \mathrm{mg} / \mathrm{dL}$ may be beneficial in high-risk patients, especially in those with multiple major risk factors who are considered to be at very high risk. ${ }^{85}$

Advances in imaging technology have also provided a better understanding of the relationship between the effects of statin therapy on dyslipidemia and the progression of atherosclerosis. While the ASTEROID study confirmed that intensive LDL-C lowering with rosuvastatin is able to induce regression of atherosclerosis in patients with established coronary artery disease, ${ }^{73,74}$ the results from METEOR expanded these findings across the disease spectrum, demonstrating that rosuvastatin can slow the progression of atherosclerosis even in asymptomatic patients with early disease and at low risk for CVD. ${ }^{79}$ Although there is not yet sufficient evidence to conclude that a reduction in the rate of progression of atherosclerosis in low-risk individuals directly translates into a reduction in clinical events, the METEOR findings do highlight the potential disease burden in an apparently "low-risk" population. 
These are important findings when considered in light of the significant residual cardiovascular risk that remains despite rigorous lipid management guidelines and effective statin treatment. In an effort to understand how further reductions in cardiovascular risk may be achieved, the JUPITER trial assessed the effects of rosuvastatin on cardiovascular events in healthy subjects without elevated LDL-C, but who had raised levels of hsCRP, a known risk factor for coronary events. ${ }^{81}$ Rosuvastatin significantly reduced the incidence of major cardiovascular events compared with placebo, even in subgroups usually considered to be at low risk (eg, Framingham risk scores $\leq 10 \%$, LDL-C $\leq 100 \mathrm{mg} / \mathrm{dL}$ ), and the trial was terminated early when the clear treatment benefit was demonstrated. These results raise a number of questions regarding the wider expansion of statin use in reducing cardiovascular risk, the role of hsCRP screening, and the balance between treatment benefits and long-term safety and cost. Nevertheless, guidelines for primary prevention will likely be reassessed in light of the JUPITER findings, as they provide a stimulus for achieving further reductions in cardiovascular risk and improvements in public health. ${ }^{100}$

\section{ACKNOWLEDGMENTS}

Drs Fabbri and Maggioni have received research support and lecture fees from Astra Zeneca. Editorial assistance in the preparation of this manuscript was provided by Andree Rose. Drs Fabbri and Maggioni would also like to thank Barbara Bartolomei Mecatti for her secretarial assistance.

\section{Disclosure}

Prior to peer review the manufacturers of rosuvastatin were offered the opportunity to comment on this article.

\section{REFERENCES}

1. Yach D, Hawkes C, Gould CL, Hofman KJ. The global burden of chronic diseases: overcoming impediments to prevention and control. JAMA. 2004;291:2616-2622.

2. Cardiovascular diseases. Fact sheet no. 317. February 2007. World Health Organization web site. Available at: www.who.int. Accessed April 2009.

3. Grundy SM, Cleeman JI, Merz CN, et al. Implications of recent clinical trials for the National Cholesterol Education Program Adult Treatment Panel III Guidelines. Circulation. 2004;110:227-239.

4. Scandinavian Simvastatin Survival Study Group. Randomised trial of cholesterol lowering in 4444 patients with coronary heart disease: the Scandinavian Simvastatin Survival Study (4S). Lancet. 1994;344:1383-1389.

5. Lipid Study Group. Prevention of cardiovascular events and death with pravastatin in patients with coronary heart disease and a broad range of initial cholesterol levels: the Long Term Intervention with Pravastatin in Ischemic Disease (LIPID) study group. N Engl J Med. 1998;339:1349-1357.

6. Heart Protection Study Collaborative Group. MRC/ BHF Heart Protection Study of cholesterol lowering with simvastatin in 20536 high-risk individuals: a randomized placebo controlled trial. Lancet. 2002;360:7-22.

7. Downs JR, Clearfield M, Weis S, et al. Primary prevention of acute coronary events with lovastatin in men and women with average cholesterol levels: results of AFCAPS/TexCAPS. Air Force/Texas Coronary Atherosclerosis Prevention Study. JAMA. 1998;279:1615-1622.

8. Sacks FM, Pfeffer MA, Moye LA, et al. Cholesterol and Recurrent Events Trial Investigators. The effect of pravastatin on coronary events after myocardial infarction in patients with average cholesterol levels. N Engl J Med. 1996;335:1001-1009.

9. Shepherd J, Cobbe SM, Ford I, et al. Prevention of coronary heart disease with pravastatin in men with hypercholesterolemia: West of Scotland Coronary Prevention Study Group. N Engl J Med. 1995;333:1301-1317.

10. Baigent C, Keech A, Kearney PM, et al. Cholesterol Treatment Trialist' (CTT) Collaborators. Efficacy and safety of cholesterol-lowering treatment: 
prospective meta-analysis of data from 90,056 participants in 14 randomised trials of statins. Lancet. $2005 ; 366: 1267-1278$.

11. Cannon CP, Steimberg BA, Murphy SA, et al. Metaanalysis of cardiovascular outcomes trials comparing intensive versus moderate statin therapy. J Am Coll Cardiol. 2006;48:438-445.

12. LaRosa JC, Grundy SM, Waters DD, et al. Treating to New Targets (TNT) Investigators. Intensive lipid lowering with atorvastatin in patients with stable coronary disease. $\mathrm{N}$ Engl J Med. $2005 ; 352: 1425-1435$.

13. De Lemos JA, Blazing MA, Wiviott SD, et al. A to $\mathrm{Z}$ Investigators. Early intensive vs a delayed conservative simvastin strategy in patients with acute coronary syndromes: phase $\mathrm{Z}$ of the A to $\mathrm{Z}$ trial. JAMA. 2004;292:1307-1316.

14. Cannon CP, Braunwald E, McCabe CH, et al. Pravastatin or Atorvastatin Evaluation and Infection Therapy-Thrombolysis In Myocardial Infarction-22 Investigators. Intensive versus moderate lipid lowering with statins after acute coronary syndrome. N Engl J Med. 2004;350:1495-1504.

15. Pedersen TR, Faergeman O, Kastelein JJ, et al. Incremental Decrease in End Points Through Aggressive Lipid Lowering (IDEAL) study group. High-dose atorvastatin versus usual-dose simvastatin for secondary prevention after myocardial infarction: the IDEAL study: a randomized controlled trial. JAMA. 2005;294:2437-2445.

16. Graham I, Atar D, Borch-Johnsen K, et al. European guidelines on cardiovascular disease prevention in clinical practice: executive summary. Fourth Joint Task Force of the European Society of Cardiology and other societies on cardiovascular disease prevention in clinical practice (constituted by representatives of nine societies and by invited experts). Eur J Cardiovasc Prev Rehabil. 2007;14(suppl. 2):E1-E40.

17. Rosensen R. Statins: can the new generation make an impression? Expert Opin Emerg Drugs. 2004;9:269-279.

18. Libby P. The forgotten majority: unfinished business in cardiovascular risk reduction. J Am Coll Cardiol. 2005;46:1225-1228.

19. Davidson $\mathrm{MH}$. Reducing residual risk for patients on statin therapy: the potential role of combination therapy. Am J Cardiol. 2005;96:3K-13K.

20. Sacks FM, Rouleau JL, Moye LA, et al. Baseline characteristics in the Cholesterol and Recurrent
Events (CARE) trial of secondary prevention in patients with average serum cholesterol levels. Am J Cardiol. 1995;75:621-623.

21. Shepherd J, Blauw GJ, Murphy MB, et al. Pravastatin in elderly individuals at risk of vascular disease (PROSPER): a randomized controlled trial. Lancet. 2002;360:1623-1630.

22. Kelley GA, Kelley KS, Tran ZV. Aerobic exercise and lipids and lipoproteins in women: a meta-analysis of randomized controlled trial. J Womens Health. 2004;13:1148-1164.

23. Kelley GA, Kelley KS. Aerobic exercise and lipids and lipoproteins in men: a meta-analysis of randomized controlled trial. J Mens Health Gend. 2006;3:61-70.

24. Yusuf S, Hawkan S, Ounpuu S, et al. INTERHEART Study Investigators. Effect of potentially modifiable risk factors associated with myocardial infarction in 52 countries (INTERHEART Study): a case-control study. Lancet. 2004;364:937-952.

25. Zalesin KC, Franklin BA, Miller WM, Peterson ED, McCullough PA. Impact of obesity on cardiovascular disease. Endocrinol Metab Clin North Am. 2008;37:663-684, ix.

26. Poirier P, Giles TD, Bray GA, et al. Obesity and cardiovascular disease: pathophysiology, evaluation, and effect of weight loss. Arterioscler Thromb Vasc Biol. 2006;26:968-976.

27. Scaglione R, Argano C, Di Chiara T, Licata G. Obesity and cardiovascular risk: the new public health problem of worldwide proportions. Expert Rev Cardiovasc Ther. 2004;2:203-212.

28. Ross R. Atherosclerosis - an inflammatory disease. N Engl J Med. 1999;340:115-126.

29. Pearson TA, Mensach GA, Alexander RW. Markers of inflammation and cardiovascular disease: application to clinical and public health practice: a statement for healthcare professionals from the Center for Disease Control and Prevention and the American Heart Association. Circulation. 2003;107:499-511.

30. Ridker PM. High-sensitivity C reactive protein: potential adjunct for global risk assessment in the primary prevention of cardiovascular disease. Circulation. 2001;103:1813-1818.

31. Ridker PM, Rifai N, Rose L, et al. Comparison of C reactive protein and low density lipoprotein cholesterol levels in the prediction of first cardiovascular events. N Engl J Med. 2002;347:1557-1565. 
32. Pai JK, Pischon T, Ma J, et al. Inflammatory markers and the risk of coronary heart disease in men and women. N Engl J Med. 2004;351:2599-2610.

33. de Ferranti SD, Rifai N. C-reactive protein: a nontraditional serum marker of cardiovascular risk. Cardiovasc Pathol. 2007;16:14-21.

34. Ridker PM, Glynn RJ, Hennekens CH. C-reactive protein: adds to the predictive value of total and HDL cholesterol in determining risk of first myocardial infarction. Circulation. 1998;97:2007-2011.

35. Ridker PM. C reactive protein and the prediction of cardiovascular events among those at intermediate risk moving an inflammatory hypothesis toward consensus. J Am Coll Cardiol. 2007;49:21292138.

36. Ridker PM, Cannon CP, Morrow D, et al. Pravastatin or Atorvastatin Evaluation and Infection Therapy - Thrombolysis in Myocardial Infarction (PROVE-IT-TIMI 22) Investigators. C-reactive protein levels and outcomes after statin therapy. N Engl J Med. 2005;352:20-28.

37. Field KM. Effect of 3-hydroxy-3-methylglutaryl coenzyme A reductase inhibitors on high-sensitivity C-reactive protein levels. Pharmacotherapy. 2005;25:1365-1377.

38. Sakkinen P, Abbott RD, Curb JD, et al. C-reactive protein and myocardial infarction. J Clin Epidemiol. 2002;55:445-451.

39. Ray KK, Cannon CP. The potential relevance of the multiple lipid independent (pleiotropic) effects of statins in the management of acute coronary syndromes. J Am Coll Cardiol. 2005;46:1425-1433.

40. Ridker PM, Rifai N, Clearfield M, et al. Measurement of C-reactive protein for the targeting of statin therapy in the primary prevention of acute coronary events. N Engl J Med. 2001;344:1959-1965.

41. Morrow DA, de Lemos JA, Sabatine MS, et al. Clinical relevance of $\mathrm{C}$-reactive protein during follow up of patients with acute coronary syndromes in the Aggrastat-to-Zocor Trial. Circulation. 2006;114:281-288.

42. Ridker PM, Morrow DA, Rose LM, et al. Relative efficacy of atorvastatin $80 \mathrm{mg}$ and pravastatin $40 \mathrm{mg}$ in achieving the dual goals of low density lipoprotein cholesterol $<70 \mathrm{mg} / \mathrm{dl}$ and C-reactive protein $<2 \mathrm{mg} / \mathrm{dl}$ : an analysis of the PROVE-IT TIMI-22 trial. J Am Coll Cardiol. 2005;45:1644-1648.
43. Gordon DJ, Rifklnd BM. High density lipoprotein the clinical implications of recent studies. N Engl J Med. 1989;321:1311-1316.

44. Assmann G, Gotto AM Jr. HDL cholesterol and protective factors in atherosclerosis. Circulation. 2004;109(suppl. 1):iii8-iii14.

45. Castell WP, Garrison RJ, Wilson PW, et al. Incidence of coronary heart disease and lipoprotein cholesterol levels: the Framingham study. JAMA. 1986;256:2835-2838.

46. Shah PK, Kaul S, Nilsson J, et al. Exploiting the vascular protective effects of high density lipoprotein and its apolipoproteins: an idea whose time for testing is coming part I. Circulation. 2001;104:23762383.

47. Badimon J, Fuster V. Regression of atherosclerotic lesions by high density lipoprotein plasma fraction in the cholesterol-fed rabbit. J Clin Invest. 1990;85:1234-1241.

48. Barter P, Gotto AM, LaRosa JC, et al. Treating to New Targets Investigators. HDL cholesterol, very low levels of LDL cholesterol and cardiovascular events. N Engl J Med. 2007;357:1301-1310.

49. Linsel Nitsche P, Tall AR. HDL as a target in the treatment of atherosclerotic cardiovascular disease. Nat Rev Drug Discov. 2005;4:193-205.

50. Chapman MJ. Therapeutic elevation of HDLcholesterol to prevent atherosclerosis and coronary artery disease. Pharmacol Ther. 2006;11:893908.

51. Barter P, Gotto AM, LaRosa JC, et al. HDL cholesterol, very low levels of LDL cholesterol and cardiovascular events. N Engl J Med. 2007;357:13011310.

52. Singh IM, Shishehbor MH, Ansell BJ. High-density lipoproteins as a therapeutic target: a systematic review. JAMA. 2007;298:786-798.

53. Hausenloy DJ, Yellon DM. Targeting residual cardiovascular risk: raising high-density lipoprotein cholesterol levels. Heart. 2008;94:706-714.

54. Nissen S, Tardiff JC, Nicholls S, et al. Effect of the torcetrapib on the progression of coronary atherosclerosis. N Engl J Med. 2007;356:1304-1316.

55. Kastelein JJ, van Leuven SI, Burgess L, et al. Effect of torcetrapib on carotid atherosclerosis in familial hypercholesterolemia. N Engl J Med. 2007;356: 1620-1630. 
56. Bots ML, Visseren FL, Evans GW, et al. RADIANCE 2 Investigators. Torcetrapib and carotid intima-media thickness in mixed dyslipidemia (RADIANCE 2 Study): a randomized double-blind trial. Lancet. 2007;370:153-160.

57. Barter PJ, Caulfield M, Eriksson M, et al. ILLUMINATE Investigators. Effects of Torcetrapib in patients at high risk for coronary events. N Engl J Med. 2007;357:2109-2122.

58. Ansell BJ, Watson KE, Fogelman AM, et al. High density lipoprotein function recent advances. J Am Coll Cardiol. 2005;46:1792-1798.

59. Ansell BJ, Fonarow GC, Fogelman AM. High density lipoprotein: is it always atheroprotective? Curr Atheroscler Rep. 2006;8:405-411.

60. Nicholls SJ. HDL: still a target for new therapies? Curr Opin Investig Drugs. 2008;9:950-956.

61. Jones P, Davidson MH, Stein EA, et al. STELLAR study group. Comparison of the efficacy and safety of rosuvastatin versus atorvastatin, simvastatin and pravastatin across doses (STELLAR Trial). Am J Cardiol. 2003;92:152-160.

62. Schuster H, Barter PJ, Stender S, et al. Effective Reductions In Cholesterol Using Rosuvastatin Therapy I study group. Effect of switching statins on achievement of lipid goals: Measuring Effective Reductions in Cholesterol Using Rosuvastatin Therapy (MERCURY I) study. Am Heart J. 2004;147:705-713.

63. Brown BG, Zhao XQ, Chait A, et al. Simvastatin and niacin, antioxidant vitamins, or the combination for the prevention of coronary disease. N Engl J Med. 2001;345:1583-1592.

64. Chapman MJ. Are the effect of statins on HDL cholesterol clinically relevant? Eur Heart J Suppl. 2004;6(suppl. C):c58-c63.

65. Nicholls SJ. Rosuvastatin and progression of atherosclerosis. Expert Rev Cardiovasc Ther. 2008;6:925-933.

66. Bots ML. Carotid intima-media thickness as a surrogate marker for cardiovascular disease in intervention studies. Curr Med Res Opin. 2006;22:2181-2190.

67. Davidson MH, Dembowski E. Atherosclerosis surrogate imaging trials come of age: for better or for worse? Curr Cardiol Rep. 2008;10:521-525.

68. Nash DT. Use of vascular ultrasound in clinical trials to evaluate new cardiovascular therapies. J Natl Med Assoc. 2008;100:222-229.
69. Kastelein JJP, de Groot E. Ultrasound imaging techniques for the evaluation of cardiovascular therapies. Eur Heart J. 2008;29:849-858.

70. Ballantyne CM. Clinical trial endpoints: angiograms, events and plaque instability. Am J Cardiol. 1998;82:5M-11M.

71. Tardif J-C, Heinonen T, Orloff D, Libby P. Vascular biomarkers and surrogates in cardiovascular disease. Circulation. 2006;113:2936-2942.

72. Nissen SE, Tuzcu EM, Schoenhagen P, et al. REVERSAL Investigators. Effect of intensive compared with moderate lipid-lowering therapy on progression of coronary atherosclerosis. A randomized controlled trial. JAMA. 2004;291:1071-1080.

73. Nissen SE, Nicholls SJ, Sipahi I, et al. ASTEROID Investigators. Effect of very high-intensity statin therapy on regression of coronary atherosclerosis: the ASTEROID trial. JAMA. 2006;295:1556-1565.

74. Ballantyne CM, Raichlen JS, Nicholls JS. ASTEROID Investigators. Effect of rosuvastatin therapy on coronary artery stenoses assessed by quantitative coronary angiography: a study to evaluate the effect of rosuvastatin on intravascular ultrasoundderived coronary atheroma burden. Circulation. 2008;117:2458-2466.

75. Nicholls SJ, Tuzcu EM, Sipahi I, et al. Statins, highdensity lipoprotein cholesterol and regression of coronary atherosclerosis. JAMA. 2007;297:499-508.

76. Amarenco P, Labreuche J, Lavallee P, Touboul PJ. Statins in stroke prevention and carotid atherosclerosis: systematic review and up-to-date meta-analysis. Stroke. 2004;35:2902-2009.

77. Smilde TJ, van Wissen S, Wollersheim $H$, et al. Effect of aggressive versus conventional lipid lowering on Atherosclerosis Progression in Familial Hypercholesterolemia (ASAP): a prospective, randomised, double-blind trial. Lancet. 2001;357:577-581.

78. Taylor AJ, Kent SM, Flaherty PJ, et al. ARBITER: Arterial Biology for the Investigation of the Treatment Effects of Reducing Cholesterol: a randomized trial comparing the effects of atorvastatin and pravastatin on carotid intima medial thickness. Circulation. 2002;106:2055-2060.

79. Crouse JR, Raichlen JS, Riley WA, et al. METEOR Study Group. Effect of rosuvastatin on progression of carotid intima-media thickness in low risk individuals with subclinical atherosclerosis. JAMA. 2007;297:1344-1353. 
80. Nicholls SJ, Raichlen JS, Ballantyne CM, et al. Intravascular ultrasound evaluation of the effect of rosuvastatin versus atorvastatin on progression of coronary atherosclerosis: design of the SATURN study. Atheroscler Suppl. 2008;9:202. Abstract PO48:746

81. Ridker PM, Danielson E, Fonseca FAH, et al. JUPITER study group. Rosuvastatin to prevent vascular events in men and women with elevated C-reactive protein. N Engl J Med. 2008;359:2195-2207.

82. Ridker PM, Danielson E, Fonseca FAH, et al. Reduction in C-reactive protein and LDL cholesterol and cardiovascular event rates after initiation of rosuvastatin: a prospective study of the JUPITER trial. Lancet. 2009;373:1175-1182.

83. Glynn RJ, Danielson E, Fonseca FAH, et al. A randomized trial of rosuvastatin in the prevention of venous thromboembolism. N Engl J Med. 2009 Mar 29. 2009;360:1851-1861.

84. Hlatky MA. Expanding the orbit of primary prevention: moving beyond JUPITER. N Engl J Med. 2008;359:2280-2282.

85. Executive Summary of the Third Report of the National Cholesterol Education Program (NCEP) Expert Panel on the Detection, Evaluation and Treatment of High Blood Cholesterol in Adults (Adult Treatment Panel III). JAMA. 2001;285:2486-2497.

86. Soran H, Durrington P. Rosuvastatin: efficacy, safety and clinical effectiveness. Expert Opin Pharmacother. 2008;9:2145-2160.

87. Kostapanos MS, Milionis HJ, Elisaf MS. An overview of the extra-lipid effects of rosuvastatin. J Cardiovasc Pharmacol Ther. 2008;13:157-174.

88. Ramasubbu K, Estep J, White DL, et al. Experimental and clinical basis for the use of statins in patients with ischemic and nonischemic cardiomyopathy. J Am Coll Cardiol. 2008;5:415-426.

89. Levy WC. Observational studies of statins in systolic heart failure. Heart Fail Clin. 2008;4:201-208.

90. Krum H, Latini R, Maggioni AP, et al. Statins and symptomatic chronic systolic heart failure: a posthoc analysis of 5010 patients enrolled in Val-HeFT. Int J Cardiol. 2007;119:48-53.
91. Kjeskus J, Apetrei E, Barrios V, et al. CORONA Group. Rosuvastatin in older patients with systolic heart failure. N Engl J Med. 2007;357:2248-2261.

92. Task Force for Diagnosis and Treatment of Acute and Chronic Heart Failure 2008 of European Society of Cardiology, Dickstein K, Cohen-Solal A, et al. ESC Guidelines for the diagnosis and treatment of acute and chronic heart failure 2008: the Task Force for the Diagnosis and Treatment of Acute and Chronic Heart Failure 2008 of the European Society of Cardiology. Developed in collaboration with the Heart Failure Association of the ESC (HFA) and endorsed by the European Society of Intensive Care Medicine (ESICM). Eur Heart J. 2008;29:2388-2442.

93. GISSI-HF Investigators, Tavazzi L, Maggioni AP, et al. Effect of rosuvastatin in patients with chronic heart failure (the GISSI-HF trial): a randomized, double-blind, placebo-controlled trial. Lancet. 2008;372:1231-1239.

94. Fornarow G. Statins and n-3 supplementation in heart failure. Lancet. 2008;37:1195-1196.

95. Selger SR, Weiss NS, Gillen DL, et al. HMG CoA reductase inhibitors are associated with reduced mortality in ESRD patients. Kidney Int. 2002;61:297-304.

96. Wanner C, Krane V, März W, et al. German Diabetes and Dialysis Study Investigators. Atorvastatin in patients with type 2 diabetes mellitus undergoing hemodialysis. N Engl J Med. 2005;353:238-248.

97. Fellström B, Zannad F, Schmieder R, et al. AURORA study group. Effect of rosuvastatin on outcomes in chronic haemodialysis patients: design and rationale of the AURORA study. Curr Control Trials Cardiovasc Med. 2005;6:9.

98. Fellström BC, Jardine AG, Schmieder RE, et al. Rosuvastatin and cardiovascular events in patients undergoing hemodialysis. $\mathrm{N}$ Engl J Med. 2009;360:1395-1407.

99. Strippoli GFM, Craig JC. Sunset for statins after AURORA. N Engl J Med. 2009;360:1455-1457.

100. Mora S, Musunuru K, Blumenthal RS. The clinical utility of high-sensitivity C-reactive protein in cardiovascular disease and the potential implication of JUPITER on current practice guidelines. Clin Chem. 2009;55:219-228. 